\title{
Bayesian Fusion Performance and System Optimization for Distributed Stochastic Gaussian Signal Detection Under Communication Constraints
}

\author{
Sudharman K. Jayaweera, Member, IEEE
}

\begin{abstract}
The problem of decentralized detection and fusion of a Gaussian signal is considered under the assumption of analog relay-amplifier local processing. It is shown that under an average global (system) power constraint, there always exists an optimal number of nodes that achieves the best possible performance under both orthogonal and nonorthogonal sensor-to-fusion center communication. Any increase in the number of nodes beyond the optimal value leads to degraded performance. This implies that each node needs to maintain a certain minimum received power level at the fusion center in order to make a useful contribution to the final decision. This is contrasted with the monotonic performance improvement observed under individual node power constraints as well as in the case of deterministic signal detection under a global power constraint. Three communication scenarios are studied in detail: 1) orthogonal; 2) equicorrelated; and 3) random signaling waveforms. In each case, error exponents and resulting bounds for Bayesian fusion performance are derived. A sensor system optimization method based on Bhattacharya error exponent, that leads to simple rules for determining the optimal number of nodes under a global average power constraint is also proposed.
\end{abstract}

Index Terms-Data fusion, distributed detection, hypothesis testing, large-system analysis, random spreading, random matrix theory, sensor networks.

\section{INTRODUCTION}

$\mathbf{T}$ HE general distributed detection problem, first posed by Tenney and Sandell in [1], involves designing optimal local and fusion center tests when only the local decisions made by remote nodes are available at a fusion center. In most cases the local processing at distributed nodes could be a form of lossy compression or simple relaying. The preliminary local processing of data at sensor nodes may be preferred due to various reasons such as capacity of the communications channel, limited processing power at the fusion center, finite average power (or energy) constraints on both sensor nodes and the fusion center, maximum peak power constraints on sensor nodes, reliability and survivability of the system. Since [1] there has

Manuscript received September 14, 2005; revised June 7, 2006. This work was supported in part by the Kansas National Science Foundation (NSF) EPSCOR program by Grants KUCR \#NSF32223/KAN32224 and KUCR \#NSF32241. This paper was presented in part at the IEEE International Conference on Acoustics, Speech and Signal Processing (ICASSP'06) Toulouse, France, May 2006. The associate editor coordinating the review of this paper and approving it for publication was Prof. Mats Viberg.

The author was with the Department of Electrical and Computer Engineering, Wichita State University, Wichita, KS, 67260 USA. He is now with the Department of Electrical and Computer Engineering, University of New Mexico, Albuquerque, NM 87131 USA (e-mail: jayaweera@ece.unm.edu).

Digital Object Identifier 10.1109/TSP.2006.888888 been a considerable amount of work on distributed detection schemes, fusion center rules as well as their performance analysis under various assumptions [2]-[5].

However, most of these investigations tend to ignore effects of noisy and bandlimited communication channel between local sensors and the fusion center. This might have been due to the traditional assumption of a relatively reliable wire-line connection, such as an optical fibre, from each sensor node to the fusion center. However, in the context of distributed wireless sensor networks that are becoming increasingly popular, such an assumption might be questionable. Due to highly dynamic nature of wireless channels it is imperative that one takes into account communication resource constraints that may result in possible communication errors. Motivated by such considerations, recently there has been a renewed interest in distributed detection under communication constraints as can be found, for example, in [6]-[12].

The two main physical resources in many communication systems, and in particular in low-power distributed wireless sensor or ad hoc networks, are power and bandwidth. There are two ways of taking the power constraints into account in a system. In the first method, each node is assumed to be subjected to an individual power constraint. A second approach suitable for certain other scenarios is to place a finite total (global) power constraint on the whole sensor system as in [7]. This implies that as number of nodes in the network increases the power available for individual nodes correspondingly decreases. This allows trading off individual node power against the number of nodes in the network and vice versa. For example, in certain applications the cost of a node may be dominated by the cost of batteries. In such situations it may be necessary to determine whether to deploy a few nodes with high power or a large number of nodes with each having low power. Also, when the sensor system is powered by a distributed power source (for example, solar power) with a certain power density per unit area the total available power may be constant justifying application of the global power model. The fusion performance for a deterministic signal under a global power-constraint was considered in [7] assuming orthogonal signaling from sensors-to-fusion center. The conclusion was that it is better to divide the available power among as many nodes as possible. In [9], it was shown that the same conclusion holds even when communication channel is band limited, leading to nonorthogonal communication between sensors and the fusion center.

This paper extends the above work by considering decentralized detection of a stochastic Gaussian signal under commu- 
nication constraints. Note that, in situations where it is difficult to characterize the structure of the signal to be detected it is common to model it as being an instance of a stochastic process. This random signal model is justifiable, for example, in case of radar-jamming signals, communication signals that are reflected from ionosphere as well as signals encountered in radio astronomy [13]. The stochastic signal model might also be applicable in certain environmental monitoring applications. This paper first derives the fusion performance under an individual node power constraint. The new contributions include fusion performance analysis with nonorthogonal sensor-to-fusion center communication especially in the limit of asymptotically large systems.

Next, the fusion performance of a random Gaussian signal in a sensor system subjected to a global power constraint is considered. It has been shown recently in [10] that for a given global power constraint on the system there is always an optimal number of nodes to be used. This is quite different from the monotonic performance improvement observed in the case of detecting a deterministic signal subjected to a global power constraint [7], [9]. The analysis in [10], however, was limited to orthogonal sensor-to-fusion center communication. In a large sensor network it may be natural to consider nonorthogonal sensor signaling. This could either be due to having only a finite bandwidth available for the whole system, thus necessitating efficient spectrum sharing via nonorthogonal signaling, or due to unavoidable practical considerations of signal distortion resulting from asynchronous system operation. Interestingly, it was shown in [14] that nonorthogonal sensor-to-fusion center communication is in fact advantageous in the case of deterministic signals. However, this possibly counter-intuitive result is due to the assumption that coherence among distributed nodes is achievable. In practice, there is a price to pay in terms of available resources in order to achieve this coherence (which is somewhat similar to the distributed beam-forming discussed in [15]). For these reasons in this paper we consider both orthogonal as well as nonorthogonal sensor-to-fusion center signaling. In particular, we investigate the following: 1) orthogonal; 2) equicorrelated; and 3) random signaling. As we will see later, only in certain situations the Bayesian fusion performance can be derived in closed form. As a result, we characterize the fusion performance via Chernoff and Bhattacharya error exponents and associated bounds [16]. This error exponent-based performance analysis shows that there is always an optimal number of nodes that leads to the best fusion performance when the system is subjected to a global power constraint. A method based on the maximization of Bhattacharya error exponent is proposed in order to optimize the fusion system performance in those situations. We show that in many cases the proposed method leads to simple rules for determining the optimal number of nodes.

In this paper, we limit ourselves to the case of distributed Bayesian detection. It should be noted that in this paper we do not address the problem of finding optimal local tests necessary for distributed detection. Rather, our interest is in analyzing the fusion performance under communication resource constraints with analog relay-amplifier local processing. This is a resource-aware distributed processing scheme in which the amplifier gain is determined by the available power at a node.
While its simplicity is probably the most attractive feature in the context of low-power sensor networks, it is perhaps also of interest to note that it has been shown to perform very well in the presence of additive noise under certain conditions [17].

The remainder of this paper is organized as follows: In Section II we present our system model and formulate the decentralized detection and fusion problem. Next, in Section III we analyze the fusion performance of a stochastic Gaussian signal under the assumption of individual node power constraints. Section IV derives the corresponding fusion performance and large system error exponents under a global system power constraint. In Sections III and IV we also present numerical examples and discuss design guidelines that emerge from our analysis. Finally, in Section V we conclude by summarizing our results and pointing out further research directions.

\section{SySTEM MODEL DESCRIPTION}

Consider a binary hypothesis testing problem in a distributed sensor system consisting of $n$-nodes and a fusion center. The null and alternative hypotheses are denoted by $H_{0}$ and $H_{1}$, respectively, having corresponding prior probabilities $P\left(H_{0}\right)=$ $\pi_{0}$ and $P\left(H_{1}\right)=\pi_{1}$. The $k$ th node observation $z_{k}$, for $k=$ $1, \ldots n$, can be written as

$$
\begin{array}{ll}
H_{0}: & z_{k}=v_{k} \\
H_{1}: & z_{k}=X_{k}+v_{k}
\end{array}
$$

where the set of observation noise samples and the set of desired signal samples are distributed as $\mathbf{v} \sim \mathcal{N}\left(\mathbf{0}, \Sigma_{v}\right)$, and $\mathbf{X} \sim \mathcal{N}\left(\mathbf{0}, \Sigma_{x}\right)$, respectively, with $\mathbf{0}$ denoting the $n$-vector of all zeros. In this paper, we limit ourselves to the case in which both $v_{k}$ s and $X_{k}$ s are independent and identically distributed (iid) sequences so that $\Sigma_{v}=\sigma_{v}^{2} \mathbf{I}$ and $\Sigma_{x}=\sigma_{x}^{2} \mathbf{I}$ where $\mathbf{I}$ is the $n \times n$ identity matrix. Moreover, the noise $\mathbf{v}$ and the signal $\mathbf{X}$ are independent of each other. We define the observation quality signal-to-noise ratio (SNR) at each node as $\gamma_{0}=\left(\sigma_{x}^{2} / \sigma_{v}^{2}\right)$.

In a distributed network, each node processes its observation $z_{k}$ independently to generate a local decision $u_{k}\left(z_{k}\right)$ and communicates it to the fusion center. The fusion center makes a final decision $u_{0}(\mathbf{r})$ where $\mathbf{r}\left(u_{1}\left(z_{1}\right), u_{2}\left(z_{2}\right), \cdots, u_{n}\left(z_{n}\right)\right)$ is the received signal at the fusion center. In general, the problem of distributed detection and fusion involves simultaneous optimization of both local and global (i.e., fusion) decision rules $u_{1}\left(z_{1}\right), u_{2}\left(z_{2}\right), \ldots, u_{n}\left(z_{n}\right), u_{0}(\mathbf{r})$. However, it is known that finding those decision rules under general conditions can be a formidable task [1], [2], [4]. Optimal local processing schemes have been derived only under certain special assumptions. One such situation is when the observations are conditionally (conditioned on the true hypothesis) independent under both hypothesis in which all decision rules can be implemented as likelihood ratio rules. Unfortunately, even in this iid observation case the optimal thresholds of the likelihood ratio tests can, in general, be different from sensor-to-sensor.

A class of important local processors are the amplify-andrelay schemes in which each node amplifies and retransmits its observation to the fusion center [7]. When local observations 
are corrupted only by additive noise, as in our formulation, amplify-and-relay local processing is known to perform fairly well. Moreover, amplify-and-relay local processing can be an attractive choice for low-power wireless sensor networks that are becoming popular [6], [8]. In this case, the local decisions sent to the fusion center are simply given by

$$
u_{k}=g_{k} z_{k} \quad \text { for } k=1, \ldots n
$$

where $g_{k}>0$ is the analog relay amplifier gain at the $k$ th node that depends on either the specified individual node power constraint $P_{k}$ or the global system power constraint $\tilde{P}_{0}$ on the whole sensor system. For simplicity, throughout this paper we assume $P_{k}=P_{0}$ and $g_{k}=g$ for all $k$. [The issue of (distributed) power allocation in which not all $g_{k} \mathrm{~s}$ are the same will be considered in a future work.]

The $k$ th sensor node is assigned a signaling waveform (code) $\mathbf{s}_{k}$ normalized such that $\mathbf{s}_{k}^{T} \mathbf{s}_{k}=1$, for $k=1, \ldots, n$. We assume that the number of degrees of freedom (DoF) in the signaling waveform to be $N$ [for example, the number of chips per symbol in a direct-sequence code-division multiple-access (DS-CDMA) system] so that $\mathbf{s}_{k}$ is a length $N$ vector. The message $u_{k}$ of the $k$ th sensor is transmitted to the fusion center over a noisy, bandlimited wireless channel by modulating onto the signaling waveform $\mathbf{s}_{k}$. Hence, $k$ th sensor's transmit signal is given by $g_{k} z_{k} \mathbf{s}_{k}$. Throughout this paper we assume an additive white Gaussian noise (AWGN) channel and ignores the effects of fading. Assuming synchronized sensor transmissions, the received chip-rate sampled signal at the fusion center can be written as

$$
\mathbf{y}=\sum_{k=1}^{n} u_{k} \mathbf{s}_{k}+\boldsymbol{\eta}
$$

where $\boldsymbol{\eta}$ is the sampled (at the chip-rate) white Gaussian receiver noise with double-sided spectral density $\sigma_{w}^{2}$ so that $\boldsymbol{\eta} \sim$ $\mathcal{N}\left(\mathbf{0}, \sigma_{w}^{2} \mathbf{I}\right)$. A sufficient statistic for the fusion center processing is obtained by passing $\mathbf{y}$ through a bank of matched-filters (each matched to a signaling waveform of a particular node) [16]. The output of the bank of matched filters can be written in vector notation as

$$
\mathbf{r}=g \mathbf{R} \mathbf{z}+\mathbf{w}
$$

where $\mathbf{z}=\left[z_{1}, \ldots, z_{n}\right]^{T}, \mathbf{w} \sim \mathcal{N}\left(\mathbf{0}, \sigma_{w}^{2} \mathbf{R}\right)$ is the $n$-dimensional, filtered noise vector and $\mathbf{R}$ is the $n \times n$, symmetric and normalized received signal correlation matrix in which the $\left(k, k^{\prime}\right)$ th element is given by $\mathbf{s}_{k}^{T} \mathbf{s}_{k^{\prime}}$. It is easy to show that $\mathbf{R}$ can be written as $\mathbf{R}=\mathbf{S}^{T} \mathbf{S}$ where $\mathbf{S}$ is an $N \times n$ matrix with $\mathbf{s}_{k}$ as its $k$ th column. In the special case of orthogonal sensor-to-fusion center communication, the received signal model (3) simplifies such that $\mathbf{R}=\mathbf{I}$.

Let us define the covariance matrix $\Sigma_{r}=g^{2} \sigma_{v}^{2} \mathbf{R}^{2}+\sigma_{w}^{2} \mathbf{R}$ and the spectral decomposition of $\mathbf{R}$ to be $\mathbf{R}=\sum_{k=1}^{n} \lambda_{k} \xi_{k} \xi_{k}^{T}$. Under the assumption that the signaling codes of the sensors are all linearly independent of each other, the set of orthonormal eigenvectors $\xi_{k}$ s forms a complete basis for $\mathbb{R}^{n}$ and $\lambda_{k} \mathrm{~s}$ are the corresponding eigenvalues. With these definitions, it is easy to show that the fusion problem can be reduced to the following hypothesis testing problem:

$$
\begin{array}{ll}
H_{0}: & \mathbf{r} \sim p_{0}(\mathbf{r})=\mathcal{N}\left(\mathbf{0}, \Sigma_{r}\right) \\
H_{1}: & \mathbf{r} \sim p_{1}(\mathbf{r})=\mathcal{N}\left(\mathbf{0}, g^{2} \sigma_{x}^{2} \mathbf{R}^{2}+\Sigma_{r}\right)
\end{array}
$$

where $p_{j}(\mathbf{r})$ is the density of $\mathbf{r}$ under the hypothesis $H_{j}$, for $j=0$ and $j=1$. It is known that the optimal (e.g., Bayesian, minimax or Neyman-Pearson) fusion center designs should then be based on the likelihood ratio $\mathcal{L}(\mathbf{r})=\left(p_{1}(\mathbf{r}) / p_{0}(\mathbf{r})\right)$. As shown in Appendix I, this likelihood ratio can be written as

$\mathcal{L}(\mathbf{r})=\exp \left(\frac{1}{2} \sum_{k=1}^{n}\left|y_{k}\right|^{2}\right) \times \prod_{k=1}^{n}\left(\frac{g^{2} \sigma_{v}^{2} \lambda_{k}+\sigma_{w}^{2}}{g^{2}\left(\sigma_{x}^{2}+\sigma_{v}^{2}\right) \lambda_{k}+\sigma_{w}^{2}}\right)^{\frac{1}{2}}$

where $Y_{1}, \ldots, Y_{n}$ are a set of independent, but in general not identically distributed, zero-mean Gaussian random variables obtained by projecting the observation vector $\mathbf{r}$ on to each of the eigenvectors $\xi_{k}$ followed by scaling (see Appendix I). If the variance of the $k$ th sample $Y_{k}$ under $H_{j}$ is $\sigma_{j, k}^{2}$, for $j=0,1$ and $k=1, \ldots, n$, then it can also be shown that (see Appendix I)

$$
\sigma_{j, k}^{2}= \begin{cases}\frac{g^{2} \sigma_{x}^{2} \lambda_{k}}{g^{2}\left(\sigma_{x}^{2}+\sigma_{v}^{2}\right) \lambda_{k}+\sigma_{w}^{2}} & \text { if } j=0 \\ \frac{g^{2} \sigma_{x}^{2} \lambda_{k}}{g^{2} \sigma_{v}^{2} \lambda_{k}+\sigma_{w}^{2}} & \text { if } j=1\end{cases}
$$

The optimal fusion decision rule is given by

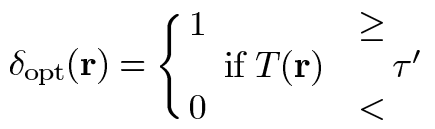

where

$$
\tau^{\prime}=2 \log \tau+\sum_{k=1}^{n} \log \left(g^{2}\left(\sigma_{x}^{2}+\sigma_{v}^{2}\right) \lambda_{k}+\sigma_{w}^{2} / g^{2} \sigma_{v}^{2} \lambda_{k}+\sigma_{w}^{2}\right)
$$

and the decision variable $T$ is the quadratic form $T(\mathbf{r})=$ $\sum_{k=1}^{n}\left|y_{k}\right|^{2}$. In (6), $\tau$ is the original threshold that depends on the exact optimality criteria. For example, for minimum probability of error Bayes detection with equal priors $\tau=1$ and in an $\epsilon$-level Neyman-Pearson design it will be determined by the false-alarm probability $\epsilon$. In this paper, however, we will only consider Bayesian optimal detectors. ${ }^{1}$ Thus, the basic performance criteria is the probability of error of a detector defined as (with equal priors)

$$
P_{e}=\frac{1}{2}\left(\alpha_{n}+\beta_{n}\right)
$$

where $\alpha_{n}$ and $\beta_{n}$ are the false-alarm and miss probabilities, respectively, in a system with $n$ sensors. However, only in certain special circumstances one can evaluate the exact probability of error $P_{e}$ of the optimal quadratic detector (6). Even when we can write the performance in closed form it may still be in terms of certain special functions that might require numerical computations. While exact, such results may fail to provide insight

\footnotetext{
${ }^{1}$ Throughout the paper, we have assumed equal priors. However, it is not difficult to modify the analysis to take into account non-equal priors. In particular, the derived optimization results hold verbatim since our error exponents do not depend on the priors.
} 
into performance trends and trade-offs. As a result, they may not lead to useful general conclusions regarding the design of decentralized sensor systems. A common method in such situations is to use error bounds and error exponents. While they may not be exact, in most situations error exponents (and the bounds based on them) can be more illuminative in characterizing the performance of a detection procedure. The most commonly used bound for Bayesian detection is the Chernoff upper bound to the probability of error which (assuming equal priors) can be written as $P_{e} \leq(1 / 2) e^{\mu_{C}}$ where Chernoff error exponent is defined as [16]

$$
\mu_{C}=\min _{s \in[0,1]} \log \mathbb{E}\left\{\mathcal{L}^{s}(\mathbf{r}) \mid H_{0}\right\} .
$$

In this paper, however, we base our analysis on the so-called Bhattacharya upper bound. Although somewhat looser than the Chernoff bound it is much easier to evaluate in situations considered in this paper. Specifically, the Bhattacharya coefficient is given by using $s=(1 / 2)$ in the expectation inside the argument of the logarithm on the right-hand side (RHS) of (8) [16]. We denote the logarithm of the Bhattacharya coefficient by $\mu_{B}$ and call it the Bhattacharya error exponent in parallel with the Chernoff error exponent defined earlier:

$$
\mu_{B}=\log \mathbb{E}\left\{\mathcal{L}^{\frac{1}{2}}(\mathbf{r}) \mid H_{0}\right\} .
$$

The Bhattacharya upper bound to the probability of error is then given by $P_{e} \leq(1 / 2) e^{\mu_{B}}$.

\section{Fusion Performance Under IndiVIDUAL NODE POWER CONSTRAINTS}

In this section, we consider the fusion performance in a system subjected to individual node power constraints. We assume that each node is subjected to the same fixed average power constraint $P_{1}=P_{2}=\cdots=P_{n}=P_{0}$ so that $\mathbb{E}\left\{\left|u_{k}\right|^{2}\right\}=P_{0}$, for all $k$. This gives the common gain factor

$$
g_{k}^{2}=g^{2} \triangleq \frac{P_{0}}{\left(\sigma_{v}^{2}+\frac{\sigma_{x}^{2}}{2}\right)}, \quad \text { for } k=1, \ldots, n .
$$

Let us also define the channel quality SNR as $\gamma_{c} \triangleq\left(P_{0} / \sigma_{w}^{2}\right)$ and, for brevity, let $\gamma=\gamma_{c} /\left(1+\left(\gamma_{0} / 2\right)\right)$.

\section{A. Orthogonal Signaling}

We first briefly consider the fusion performance of the distributed sensor system with orthogonal communication from sensors to the fusion center. Under orthogonal signaling, which requires $n \leq N$, the normalized cross-correlation matrix becomes $\mathbf{R}=\mathbf{I}$ so that all eigenvalues in (4) degenerate such that $\lambda_{k}=1$ for all $k=1, \ldots, n$. As a result, the variances $\sigma_{0, k}^{2}=\sigma_{0}^{2}$ and $\sigma_{1, k}^{2}=\sigma_{1}^{2}$ for all $k$ in (5). Using the definitions of $\gamma_{c}$ and $\gamma$ we may write them as

$$
\sigma_{j}^{2}=\left\{\begin{array}{ll}
\frac{\gamma_{0}}{1+\gamma_{0}+\frac{1}{\gamma}}, & \text { if } j=0 \\
\frac{\gamma_{0}}{1+\frac{1}{\gamma}}, & \text { if } j=1
\end{array} .\right.
$$

Similarly, the threshold $\tau^{\prime}$ simplifies to $\tau^{\prime}=n \log (1+$ $\left.\left(\gamma_{c} \gamma_{0} / 1+\left(\gamma_{0} / 2\right)+\gamma_{c}\right)\right)$. Following standard techniques it is straightforward to show that in this case $T$ is a Gamma random variable of the form of $T \sim G\left((n / 2),\left(1 / 2 \sigma_{j}^{2}\right)\right)$ under the hypotheses $H_{j}$. The probability of error of the detector (6) can then easily be shown to be [16]

$$
P_{e}=\frac{1}{2}\left(1-\frac{\Gamma\left(\frac{n}{2} ; \frac{\tau^{\prime}}{2 \sigma_{0}^{2}}\right)-\Gamma\left(\frac{n}{2} ; \frac{\tau^{\prime}}{2 \sigma_{1}^{2}}\right)}{\Gamma\left(\frac{n}{2}\right)}\right)
$$

where $\Gamma(a)=\int_{0}^{\infty} e^{-y} y^{a-1} d y$ is the Gamma function and $\Gamma(a, t)=\int_{0}^{t} e^{-y} y^{a-1} d y$ is the incomplete Gamma function. Investigation of (12) shows that the $P_{e}$ monotonically improves with increasing $n$ for all $\gamma_{0}$ and $\gamma_{c}$. This is of course well-known in classical random signal detection (see, for example, [16]): Including more node measurements always helps improve the performance. To be complete, in the following proposition we state without proof the corresponding Chernoff and Bhattacharya error exponents for the orthogonal signaling.

Proposition 1: The Chernoff and Bhattacharya error exponents with orthogonal signaling, $\mu_{C}^{0}$ and $\mu_{B}^{0}$, respectively, corresponding to the Bayesian fusion performance are given by

and

$$
\mu_{C}^{0}=\frac{n}{2}\left[\log \frac{1+\sigma_{1}^{2}}{1+\left(1-s_{0}\right) \sigma_{1}^{2}}-s_{0} \log \left(1+\sigma_{1}^{2}\right)\right]
$$

$$
\mu_{B}^{0}=\frac{n}{2}\left[\frac{1}{2} \log \left(1+\sigma_{1}^{2}\right)-\log \left(1+\frac{\sigma_{1}^{2}}{2}\right)\right]
$$

where $\sigma_{1}^{2}$ is given by (11) and $s_{0}=1+\left(1 / \sigma_{1}^{2}\right)-$ $\left(1 / \log \left(1+\sigma_{1}^{2}\right)\right)$ in $(13)$.

It can be shown that for all $\sigma_{1}^{2}>0$, the above $\mu_{C}^{0}<0$ independent of the value of $n$ indicating the monotonic performance improvement with increasing $n$. (We omit the details here but it is not difficult to establish using standard techniques.)

\section{B. Equicorrelated Signaling}

In practice, the orthogonal signaling model may not be justified due to various reasons. Even if one could assign a set of orthogonal signaling waveforms for all nodes, it is likely that due to channel dynamics this orthogonality will be lost at the receiver. In a low-power sensor network the nodes might not also be perfectly synchronized. Further, in a bandwidth-constrained, large sensor network it might be impossible to provide all nodes with orthogonal waveforms. If we assume that each node $k$ is assigned a length $N$ code $\mathbf{s}_{k}$ for channel access, the number of orthogonal codes available will be limited to $N$ where the largest possible value of $N$ will ultimately be limited by the available communication bandwidth. This makes it important to investigate the fusion performance of a distributed sensor system, in general, under nonorthogonal sensor-to-fusion center communications.

A commonly used nonorthogonal signaling model is the equicorrelation model in which correlation between any two 
different signaling waveforms is assumed to be the same, so that

$$
\mathbf{R}=\left[\begin{array}{ccccc}
1 & \rho & \rho & \cdots & \rho \\
\rho & 1 & \rho & \cdots & \rho \\
\vdots & \vdots & \vdots & \ddots & \vdots \\
\rho & \rho & \rho & \cdots & 1
\end{array}\right]
$$

where $|\rho|<1$ is the common correlation between any pair of received signaling waveforms (in the following analysis we will assume that $0<\rho<1$. The analysis for negative $\rho$ follows easily). While (15) may seem somewhat artificial this model does provide a good approximation for certain communication scenarios. For example, in the case of Gold codes [18] and the recently proposed min-TSC (total-squared-correlation) codes [19], the cross-correlation between any two distinct waveforms is guaranteed to be bounded. Moreover, in most situations of practical interest the waveform correlations may be very small although not identically zero. In those cases, small variations in different correlations may be ignored.

The eigenvalues of (15) can easily shown to be $\lambda_{1}=\lambda_{2}=$ $\cdots=\lambda_{n-1}=1-\rho \triangleq \lambda_{a}$ and $\lambda_{n}=1+(n-1) \rho \triangleq \lambda_{b}$. As a result, the decision statistic $T(\mathbf{r})$ is a sum of squares of $(n-1)$ identical zero-mean Gaussian random variables plus the square of an additional zero-mean Gaussian random variable with a different variance (all of them independent). The deviation in this one sample, however, makes the closed form analysis of the error probability significantly difficult as we see from the following proposition.

Proposition 2: The false-alarm and the miss probabilities, $\alpha_{n}$ and $\beta_{n}$ respectively, of the optimal fusion detector in distributed stochastic Gaussian signal detection with equicorrelated sensor-to-fusion center signaling are

$$
\begin{aligned}
& \alpha_{n}=\int_{\tau^{\prime \prime}}^{\infty} p_{T}\left(t \mid H_{0}\right) d t \\
& \beta_{n}=\int_{0}^{\tau^{\prime \prime}} p_{T}\left(t \mid H_{1}\right) d t
\end{aligned}
$$

where $\tau^{\prime \prime}=(n-1) \log \left(1+\sigma_{1, a}^{2}\right)+\log \left(1+\sigma_{1, b}^{2}\right)$

$$
\begin{aligned}
p_{T}\left(t \mid H_{j}\right) & \frac{\exp \left(\frac{-t}{2 \sigma_{j, b}^{2}}\right) t^{\frac{n}{2}-1}}{\Gamma\left(\frac{n}{2}\right)\left(2 \sigma_{j, a}^{2}\right)^{\frac{n-1}{2}}\left(2 \sigma_{j, b}^{2}\right)^{\frac{1}{2}}}{ }_{1} F_{1} \\
& \cdot\left(\frac{n-1}{2} ; \frac{n}{2} ;-t\left(\frac{1}{2 \sigma_{j, a}^{2}}-\frac{1}{2 \sigma_{j, b}^{2}}\right)\right)
\end{aligned}
$$

and $\sigma_{1, a}^{2}$ and $\sigma_{1, b}^{2}$ are defined in (46). In (18), ${ }_{1} F_{1}(x ; y ; t)$ is the confluent hypergeometric function defined as ${ }_{1} F_{1}(x ; y ; t)=$ $\sum_{k=0}^{\infty}\left((x)_{k} /(y)_{k}\right)\left(t^{k} / k !\right)$ where $(x)_{k}=x(x+1)(x+$
2) $\cdots(x+k-1)=(\Gamma(x+k) / \Gamma(x))$ is the Pochhammer symbol.

For $n \gg 1$, the above false-alarm and miss probabilities can be approximated as

$$
\alpha_{n} \approx 1-\frac{\Gamma\left(\frac{n}{2} ; \frac{\tau^{\prime \prime}}{2 \sigma_{0, a}^{2}}\right)}{\Gamma\left(\frac{n}{2}\right)} \text { and } \beta_{n} \approx \frac{\Gamma\left(\frac{n}{2} ; \frac{\tau^{\prime \prime}}{2 \sigma_{1, a}^{2}}\right)}{\Gamma\left(\frac{n}{2}\right)} .
$$

Proof: See Appendix II.

Substitution of (16) and (17) in (7) gives the exact fusion probability of error. To the best of our knowledge there does not exist simple closed forms for either of the integrals involving the confluent hypergeometric function in (16) or (17). Thus, for evaluating (16) or (17) we need to resort to numerical integration. A reasonable approximation to the fusion probability of error can be obtained by using (19), especially when the system size is large. As an alternative, we can consider error exponents and the related bounds. The following proposition states the Chernoff and Bhattasharya error exponents for this case:

Proposition 3: The Chernoff error exponent for Bayesian fusion performance in distributed stochastic Gaussian signal detection with equicorrelated sensor-to-fusion center signaling is

$$
\begin{aligned}
\mu_{C}= & \frac{n-1}{2}\left[\left(1-s_{0}\right) \log \left(1+\sigma_{1, a}^{2}\right)\right. \\
& \left.-\log \left(1+\left(1-s_{0}\right) \sigma_{1, a}^{2}\right)\right] \\
& +\frac{1}{2}\left[\left(1-s_{0}\right) \log \left(1+\sigma_{1, b}^{2}\right)\right. \\
& \left.-\log \left(1+\left(1-s_{0}\right) \sigma_{1, b}^{2}\right)\right]
\end{aligned}
$$

where [see (20) at the bottom of the page] with

$$
\begin{aligned}
& K_{1}=\left(4 K_{2} / K_{3}\left(1+\left(1 / \sigma_{1, b}^{2}\right)-\bar{s}_{0}\right)^{2}\right) \\
& K_{2}=\left(1 / \sigma_{1, a}^{2}\right)-\left(1 / \sigma_{1, b}^{2}\right) \\
& K_{3}=(n-1) \log \left(1+\sigma_{1, a}^{2}\right)+\log \left(1+\sigma_{1, b}^{2}\right)
\end{aligned}
$$

and $\bar{s}_{0}=1+\left(1 / \sigma_{1, a}^{2}\right)-\left(n / K_{3}\right)$. The corresponding Bhattacharya error exponent can be written as

$$
\begin{aligned}
\mu_{B}=\frac{n-1}{2} & {\left[\frac{1}{2} \log \left(1+\sigma_{1, a}^{2}\right)-\log \left(1+\frac{\sigma_{1, a}^{2}}{2}\right)\right] } \\
+ & \frac{1}{2}\left[\frac{1}{2} \log \left(1+\sigma_{1, b}^{2}\right)-\log \left(1+\frac{\sigma_{1, b}^{2}}{2}\right)\right] .
\end{aligned}
$$

Note that when $\rho=0$ we have $s_{0}=\bar{s}_{0}$ in (20) (since in this case $K_{2}=K_{1}=0$ due to $\sigma_{1, a}^{2}=\sigma_{1, b}^{2}$ ). Fig. 1 shows the exact error probability computed via the numerical integration of the pdf (19) and the large $n$ approximation given in (19). Clearly, the difference from the exact performance is negligible unless $n$ is very small. Although approximation worsens as $\rho$ increases, for sufficiently large $n$ the approximation becomes closer to the true error probability even for large $\rho$ values. Fig. 1 also shows that signaling correlations always tend to degrade the performance. From (19) note that the difference in

$$
s_{0}= \begin{cases}\frac{1}{2}\left[\bar{s}_{0}\left(1+\sqrt{1+K_{1}}\right)-\left(1+\frac{1}{\sigma_{1, b}^{2}}\right)\left(\sqrt{1+K_{1}}-1\right)\right] & \text { if } \bar{s}_{0} \leq 1+\frac{1}{\sigma_{1, b}^{2}} \\ \frac{1}{2}\left[\bar{s}_{0}\left(1-\sqrt{1+K_{1}}\right)+\left(1+\frac{1}{\sigma_{1, b}^{2}}\right)\left(\sqrt{1+K_{1}}+1\right)\right] & \text { otherwise }\end{cases}
$$




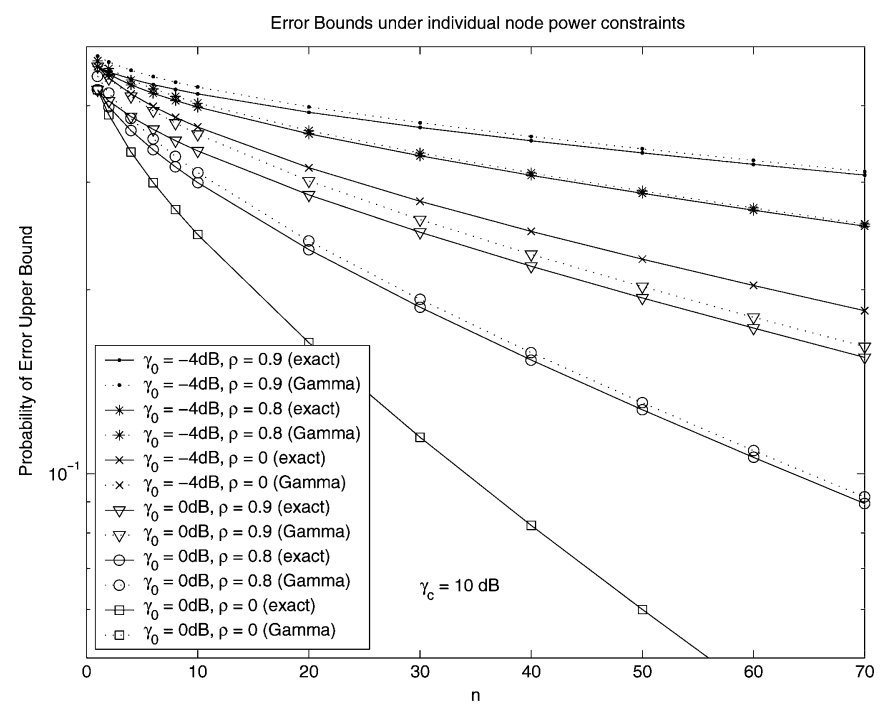

Fig. 1. Fusion probability of error with equicorrelated signaling under individual node power constraints.

performance from the orthogonal communication essentially stems due to the replacement of the ratios $\left(\tau^{\prime} / 2 \sigma_{j}^{2}\right)$ in (12) with $\left(\tau^{\prime \prime} / 2 \sigma_{j, a}^{2}\right)$. However, for $n \gg 1$ it can be seen that $\left(\tau^{\prime \prime} / 2 \sigma_{j, a}^{2}\right) \approx\left(n \log \left(1+\left(\gamma_{0} / 1+\left(1 / \gamma \lambda_{a}\right)\right)\right) / 2 \sigma_{j, a}^{2}\right)$. Thus, a comparison with the orthogonal signaling reveals that the performance is essentially as if individual node power constraints were $(1-\rho) P_{0}$ rather than the actual value $P_{0}$, at least when $n$ is large. This shows that the effect of nonorthogonal signaling always degrades the final fusion performance. The larger the signaling waveform correlation $\rho$ the more the performance will degrade as one would expect intuitively. On the other hand, including more nodes always leads to better performance regardless of the value of $\rho$.

The performance upper bounds obtained via Chernoff and Bhattacharya exponents along with the large $n$ approximation to error probability given in terms of the Gamma distribution are shown in Fig. 2. It can be shown that for small $\gamma_{0}$ the optimal $s_{0} \longrightarrow 0.5$, thus making the Bhattacharya exponent as good as the Chernoff exponent. Similarly, it can be shown that for large $n$ and large $\gamma_{0}$

$$
s_{0} \longrightarrow 1+\frac{1}{2(1-\rho) \gamma_{c}}-\frac{1}{\log \left(1+2(1-\rho) \gamma_{c}\right)} .
$$

Thus the Bhattacharya bound is expected to be loose for large $\gamma_{0}$ compared to that of Chernoff bound. Note that for sufficiently large $n$, the optimal $s_{0}(20)$ that results in the tightest upper bound of the Chernoff-type decreases with increasing $\rho$. Hence, this deviation is worst for smaller values of $\rho$. This is clear from Fig. 2.

\section{Under Random Signaling}

Another widely used model, especially popular in DS-CDMA spread spectrum communication performance analysis literature, is the random signaling waveform (code) model. Here it is assumed that the transmit signaling waveform $\mathbf{s}_{k}$ of the $k$ th sensor is a pseudo-random code of length $N$ in which each element is an independent and identically distributed random variable with zero-mean and variance $(1 / N)$, where $N$ is the

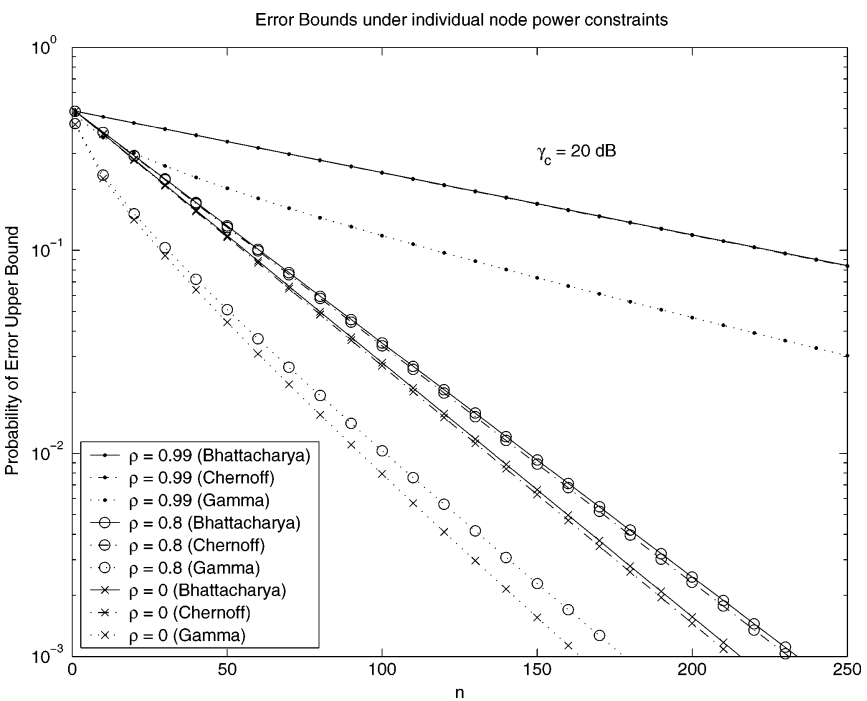

Fig. 2. The upper bounds and approximation for the fusion probability of error with equicorrelated signaling under individual node power constraints.

number of degrees of freedom (for example, number of chips per symbol in a DS-CDMA system). For example, we may consider the sequences as binary with each element being either $\pm(1 / \sqrt{N})$ with equal probability although our results hold under more general conditions [20], [21]. It is also assumed that $\mathbf{s}_{k}$ s are chosen independently so that the $N \times n$ matrix of transmit waveforms $\mathbf{S}=\left[\mathbf{s}_{1}, \mathbf{s}_{2}, \ldots, \mathbf{s}_{n}\right]$ is a random matrix of iid elements. As aforementioned, the fusion performance depends on the matrix $\mathbf{R}=\mathbf{S}^{T} \mathbf{S}$ only through its eigenvalues, which in this case are themselves random. For simplicity, in the following we concentrate on Bhattacharya error exponent and the resulting fusion probability of error bound. The key results are summarized below.

Proposition 4: Consider a large sensor system with random signaling in which dimensions $n$ and $N$ grows without bound such that $\lim _{n \longrightarrow \infty}(n / N)=\beta$. The normalized Bhattacharya error exponent corresponding to the fusion error probability of a Gaussian signal with random signaling waveforms converges almost surely (a.s.) in the limit of a large system to (23), shown at the bottom of the next page, where we have defined $\gamma_{1}=$ $\gamma, \gamma_{2}=\left(1+\gamma_{0}\right) \gamma, \gamma_{3}=\left(1+\left(\gamma_{0} / 2\right)\right) \gamma$ and

$$
\psi(x, \lambda) \triangleq(\sqrt{x b(\lambda)+1}-\sqrt{x a(\lambda)+1})^{2}
$$

with

$$
a(\lambda)=(1-\sqrt{\lambda})^{2} \quad \text { and } \quad b(\lambda)=(1+\sqrt{\lambda})^{2} .
$$

Moreover, when $\gamma_{0} \gg 1$ the Bhattacharya error exponent in a large system can be written as

$$
\begin{aligned}
\mu_{B} \approx \frac{n}{2}[ & \log \frac{\sqrt{\left(1+2 \gamma_{c}-\frac{1}{4} \psi\left(2 \gamma_{c}, \beta\right)\right)}}{1+\gamma_{c}-\frac{1}{4} \psi\left(\gamma_{c}, \beta\right)} \\
& +\frac{1}{\beta} \log \frac{\sqrt{\left(1+2 \gamma_{c} \beta-\frac{1}{4} \psi\left(2 \gamma_{c}, \beta\right)\right)}}{1+\gamma_{c} \beta-\frac{1}{4} \psi\left(\gamma_{c}, \beta\right)} \\
& \left.-\frac{1}{16 \beta \gamma_{c}}\left(\psi\left(2 \gamma_{c}, \beta\right)-4 \psi\left(\gamma_{c}, \beta\right)\right)\right] .
\end{aligned}
$$


Proof: See Appendix III.

Note that, (26) asserts that when observation quality at sensor nodes are good the fusion performance will only be limited by the communication channel quality $\gamma_{c}$. We also have following two immediate corollaries.

Corollary 1: Regardless of the value of $n$

$$
\lim _{\beta \longrightarrow 0} \mu_{B}=\mu_{B}^{0}
$$

where $\mu_{B}^{0}$ denotes the Bhattacharya error exponent with orthogonal signaling.

Proof: It can be verified that, $\lim _{\beta \longrightarrow 0} \psi(x, \beta)=0$ and

$$
\lim _{\beta \longrightarrow 0}\left[\frac{1}{2} \frac{\log \left(1+x \beta-\frac{\psi(x, \beta)}{4}\right)}{\beta}-\frac{\psi(x, \beta)}{8 x \beta}\right]=0 .
$$

Using these in taking the limit $\beta \longrightarrow 0$ of (23), we obtain the same expression as that of $\mu_{B}^{0}$ given in (14).

Since $\beta=\lim _{n \longrightarrow \infty}(n / N)$, essentially $\beta \longrightarrow 0$ implies that $N \gg n$. In other words, there are many more DoFs in signaling compared to the number of sensor nodes, meaning that it is possible provide orthogonal signaling to all nodes. Note that, in light of this the conclusion (27) of Corollary 1 is immediate.

Corollary 2: In the special case of $\beta=1$, the normalized Bhattacharya exponent converges almost surely to

$$
\begin{aligned}
\frac{\mu_{B}}{n} \stackrel{\text { a.s. }}{\rightarrow} \frac{1}{2}[ & \log \frac{\left(1+\sqrt{4 \gamma_{1}+1}\right)\left(1+\sqrt{4 \gamma_{2}+1}\right)}{\left(1+\sqrt{4 \gamma_{3}+1}\right)^{2}} \\
& +\frac{\left(\sqrt{4 \gamma_{3}+1}-1\right)^{2}}{4 \gamma_{3}}-\frac{\left(\sqrt{4 \gamma_{1}+1}-1\right)^{2}}{8 \gamma_{1}} \\
& \left.-\frac{\left(\sqrt{4 \gamma_{2}+1}-1\right)^{2}}{8 \gamma_{2}}\right] .
\end{aligned}
$$

Proof: When $\beta=1$, integral (51) in Appendix IV simplifies to

$$
\begin{aligned}
\int_{0}^{\infty} & \log (1+x \lambda) f(\lambda) d \lambda \\
= & 2 \log (1+\sqrt{4 x+1})-\frac{(\sqrt{4 x+1}-1)^{2}}{4 x} \\
& -2 \log (2) .
\end{aligned}
$$

Substituting this in (23) leads to (29).

Fig. 3 shows the exact (random) and asymptotic Bhattachrya error exponents. It is interesting to note that the asymptotic error exponent in fact is tight even for relatively small values of $n$. Under individual node power constraints the probability of fusion error monotonically improves with increasing $n$ as one would have expected. Fig. 3 also shows the performance penalty

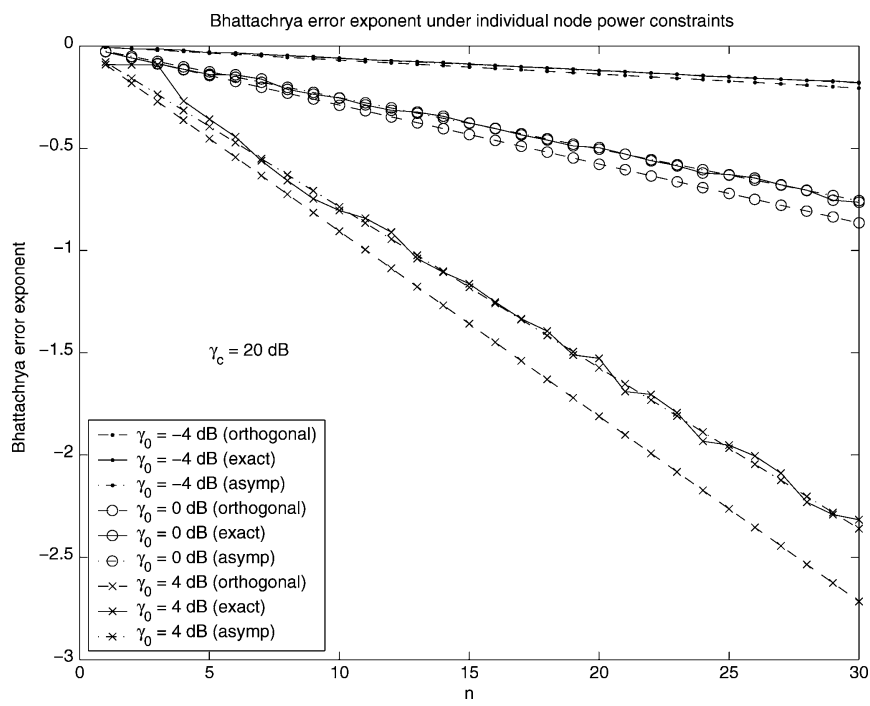

Fig. 3. The large system approximation to the Bhattacharya error exponent with random signaling under individual node power constraints.

due to the use of nonorthogonal sensor-to-fusion center communication via random waveforms. The performance degradation in terms of the Bhattacharya error exponent (and thus in terms of fusion error probability) monotonically increases with increasing $n$. However, it can be shown that the normalized performance penalty defined as $\Delta \mu_{B}=\left(\mu_{B}-\mu_{B}^{0}\right) / n$ is independent of $n$, for large $n$. This is of course a consequence of the convergence of the empirical distribution of the eigenvalues. On the other hand $\Delta \mu_{B}$ is a function of the observation SNR quality. Indeed it can be proven that the normalized performance penalty $\Delta \mu_{B}$ increases with $\gamma_{0}$ before finally converging to

$$
\begin{aligned}
\lim _{\gamma_{0} \rightarrow \infty} \Delta \mu_{B} & \stackrel{\text { a.s. }}{\longrightarrow} \frac{1}{2}\left[\log \frac{2\left(1+\gamma_{c}\right)\left(1+\sqrt{8 \gamma_{c}+1}\right)}{\sqrt{1+2 \gamma_{c}}\left(1+\sqrt{4 \gamma_{c}+1}\right)^{2}}\right. \\
& \left.+\frac{\left(\sqrt{4 \gamma_{c}+1}-1\right)^{2}}{4 \gamma_{c}}-\frac{\left(\sqrt{8 \gamma_{c}+1}-1\right)^{2}}{16 \gamma_{c}}\right] .
\end{aligned}
$$

\section{Fusion Performance Under a TOtal Average POWER CONSTRAINT}

Let us now consider a system subjected to a global average power constraint $\tilde{P}_{0}$. In this case we can show that the amplifier gain is given by

$$
g^{2}=\tilde{g}^{2} \triangleq \frac{\tilde{P}_{0}}{n\left(\sigma_{v}^{2}+\frac{\sigma_{x}^{2}}{2}\right)} .
$$

Note that, as more nodes are introduced the gain at each node correspondingly decreases. Let us also redefine the channel

$$
\begin{aligned}
& \frac{\mu_{B}}{n} \stackrel{\text { a.s. }}{\rightarrow} \frac{1}{2}\left[\log \frac{\sqrt{\left(1+\gamma_{1}-\frac{1}{4} \psi\left(\gamma_{1}, \beta\right)\right)\left(1+\gamma_{2}-\frac{1}{4} \psi\left(\gamma_{2}, \beta\right)\right)}}{1+\gamma_{3}-\frac{1}{4} \psi\left(\gamma_{3}, \beta\right)}\right. \\
& \left.+\frac{1}{\beta} \log \frac{\sqrt{\left(1+\gamma_{1} \beta-\frac{1}{4} \psi\left(\gamma_{1}, \beta\right)\right)\left(1+\gamma_{2} \beta-\frac{1}{4} \psi\left(\gamma_{2}, \beta\right)\right)}}{1+\gamma_{3} \beta-\frac{1}{4} \psi\left(\gamma_{3}, \beta\right)}-\frac{1}{8 \beta}\left(\frac{\psi\left(\gamma_{1}, \beta\right)}{\gamma_{1}}+\frac{\psi\left(\gamma_{2}, \beta\right)}{\gamma_{2}}-\frac{2 \psi\left(\gamma_{3}, \beta\right)}{\gamma_{3}}\right)\right]
\end{aligned}
$$


quality SNR as $\gamma_{c} \triangleq\left(\tilde{P}_{0} / \sigma_{w}^{2}\right)$ and, $\tilde{\gamma}=\left(\gamma_{c} / n\left(1+\left(\gamma_{0} / 2\right)\right)\right)$. In the following, we investigate fusion probability of error under a total system power constraint for the same three situations considered earlier.

\section{A. Under Orthogonal Signaling}

The exact fusion error probability and its asymptotic behavior under orthogonal signaling is summarized in the following proposition:

Proposition 5: The minimum error probability achieved by the optimal Bayesian detector for a random Gaussian signal is given by (assuming equal priors)

$$
P_{e}=\frac{1}{2}\left[1+\frac{\Gamma\left(\frac{n}{2} ; \frac{\tau^{\prime}}{2 \tilde{\sigma}_{1}^{2}}\right)-\Gamma\left(\frac{n}{2} ; \frac{\tau^{\prime}}{2 \tilde{\sigma}_{0}^{2}}\right)}{\Gamma\left(\frac{n}{2}\right)}\right]
$$

where $\tau^{\prime}=\tilde{\tau}^{\prime}=n \log \left(1+\left(\gamma_{c} \gamma_{0} / n\left(1+\left(\gamma_{0} / 2\right)\right)+\gamma_{c}\right)\right), \tilde{\sigma}_{0}^{2} \triangleq$ $\left(\gamma_{0} / 1+\gamma_{0}+(1 / \tilde{\gamma})\right)$ and $\tilde{\sigma}_{1}^{2} \triangleq\left(\gamma_{0} / 1+(1 / \tilde{\gamma})\right)$. Moreover, it exhibits the following asymptotic properties:

$$
\begin{aligned}
\lim _{n \rightarrow \infty} P_{e} & =0.5 \\
\lim _{\gamma_{0} \longrightarrow \infty} P_{e} & =\frac{1}{2}\left(1-\frac{\Gamma\left(\frac{n}{2} ; t_{0}\right)-\Gamma\left(\frac{n}{2} ; t_{1}\right)}{\Gamma\left(\frac{n}{2}\right)}\right)
\end{aligned}
$$

where $t_{0}=t_{1}+(n / 2) \log \left(1+\left(\gamma_{c} /(n / 2)\right)\right)$ and $t_{1}=$ $\left(n^{2} / 4 \gamma_{c}\right) \log \left(1+\left(\gamma_{c} /(n / 2)\right)\right)$.

The key observation from Proposition 5 is that, in contrast to the case of individual node power constraints, the final fusion performance is not monotonic in the number of nodes $n$. In fact, as can be seen from Fig. 4(a), there is an optimal number of sensor nodes for each $\gamma_{0}$ and $\gamma_{c}$ combination, beyond of which the performance monotonically degrades. Although this behavior was observed in [10], it did not address the issue of deriving the optimal number of nodes, partly because the error probability expression as given in (33) is too complicated to be useful for that purpose. Fig. 4(b) shows the convergence of fusion probability of error to the asymptotic bound (35) given in Proposition 5 for large $\gamma_{0}$ values.

In order to investigate the optimal number of nodes to be used in this scenario, we resort to the error exponents. By modifying Proposition 1, it can be shown that the Chernoff and Bhattacharya exponents are given by

$$
\begin{aligned}
& \mu_{C}=\frac{n}{2}\left[\log \frac{1+\tilde{\sigma}_{1}^{2}}{1+\left(1-s_{0}\right) \tilde{\sigma}_{1}^{2}}-s_{0} \log \left(1+\tilde{\sigma}_{1}^{2}\right)\right] \\
& \mu_{B}=\frac{n}{2}\left[\frac{1}{2} \log \left(1+\tilde{\sigma}_{1}^{2}\right)-\log \left(1+\frac{\tilde{\sigma}_{1}^{2}}{2}\right)\right]
\end{aligned}
$$

where $s_{0}=1+\left(1 / \tilde{\sigma}_{1}^{2}\right)-\left(1 / \log \left(1+\tilde{\sigma}_{1}^{2}\right)\right)$ in (36). Interestingly, using the fact that $\tilde{\sigma}_{1}^{2} \ll 1$ for $n \gg 1$, we can show that $\lim _{n \rightarrow \infty} \mu_{B}=0$. This indicates that the Bhattacharya upper bound to the error probability goes to 0.5 hinting that the fusion error may also degrade in large systems. Fig. 4(a) also shows the behavior of $\mu_{B}$ as a function of $n$ for a fixed $\gamma_{c}$. (We have not included $\mu_{C}$ since it is very close to $\mu_{B}$ for the considered range of $\gamma_{0}$ and $\gamma_{c}$ values.) Clearly there are optimal values of $n$ for which both $\mu_{B}$ and $\mu_{C}$ are minimized. Moreover, although the bound could be somewhat loose, the optimal $n=n_{0}$ for
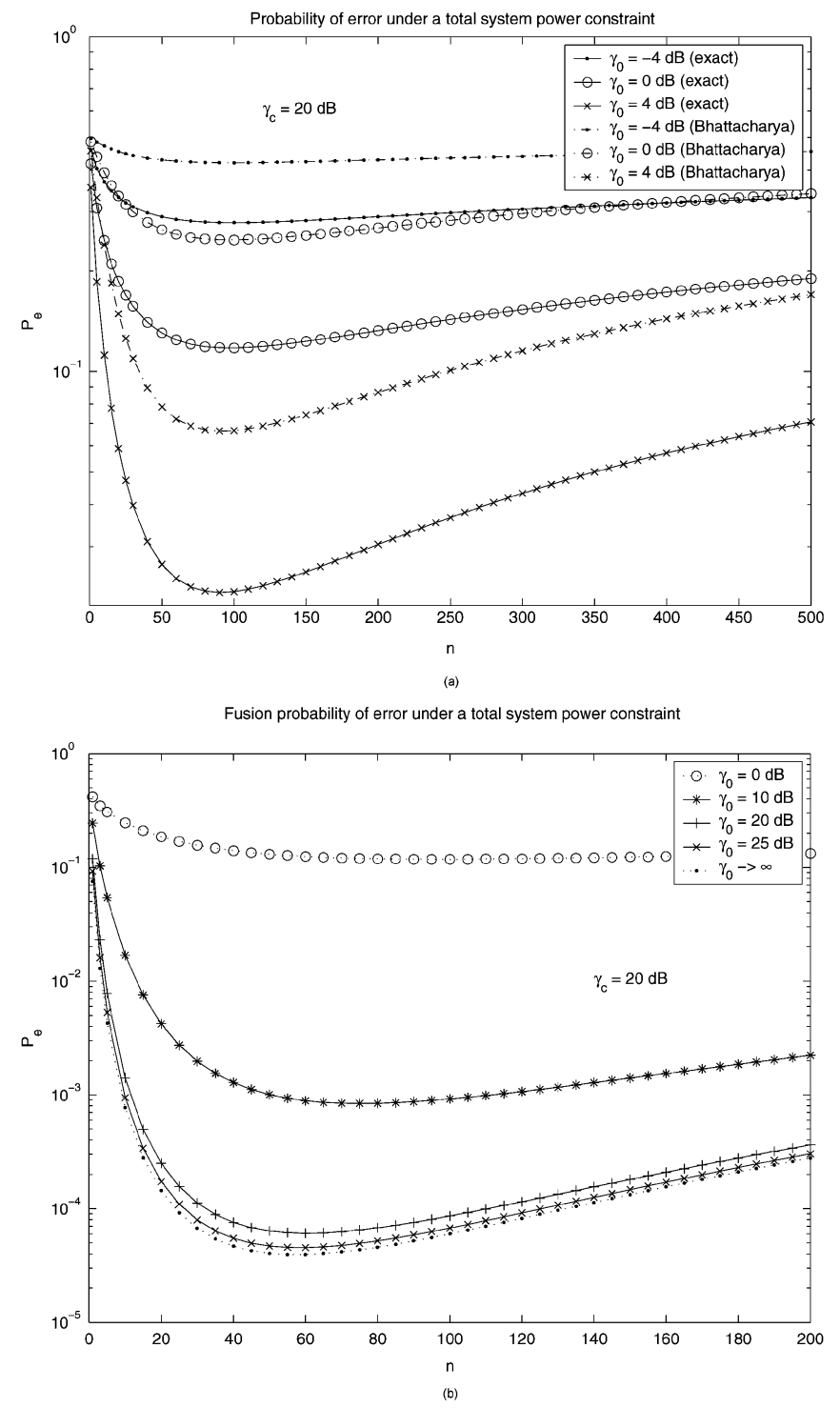

Fig. 4. Minimum achievable fusion probability of error in distributed detection of a random signal with orthogonal sensor-to-fusion center communication under a global system power constraint. $\gamma_{c}=20 \mathrm{~dB}$. (a) Exact error probability and Bhattacharya upper-bound. (b) Limiting behavior for large $\gamma_{0}$.

Bhattacharya bound seems to be almost the same as that for the exact error probability. This motivates us to use the Bhattacharya exponent as the basis for optimizing the sensor system size due to its relative simplicity. The following proposition summarizes the solution to the sensor system optimization problem in the case of orthogonal signaling under a global power constraint.

Proposition 6: The optimal number of nodes $n_{0}$ that results in the minimum Bhattacharya upper bound to the fusion error probability in a distributed sensor system subjected to a global power constraint $\tilde{P}_{0}$ is given by

$$
n_{0}=\frac{\gamma_{c}}{\left(\frac{1}{2}+\frac{1}{\gamma_{0}}\right)}\left(\frac{1}{2 x_{0}}-\frac{1}{\gamma_{0}}\right)
$$

where $x=x_{0}$ is the unique positive solution to the equation $f_{\gamma_{0}}(x)=0$ with

$$
f_{\gamma_{0}}(x)=\log \frac{\sqrt{1+2 x}}{1+x}+\left(1-\frac{2 x}{\gamma_{0}}\right) \frac{x^{2}}{(1+x)(1+2 x)} .
$$



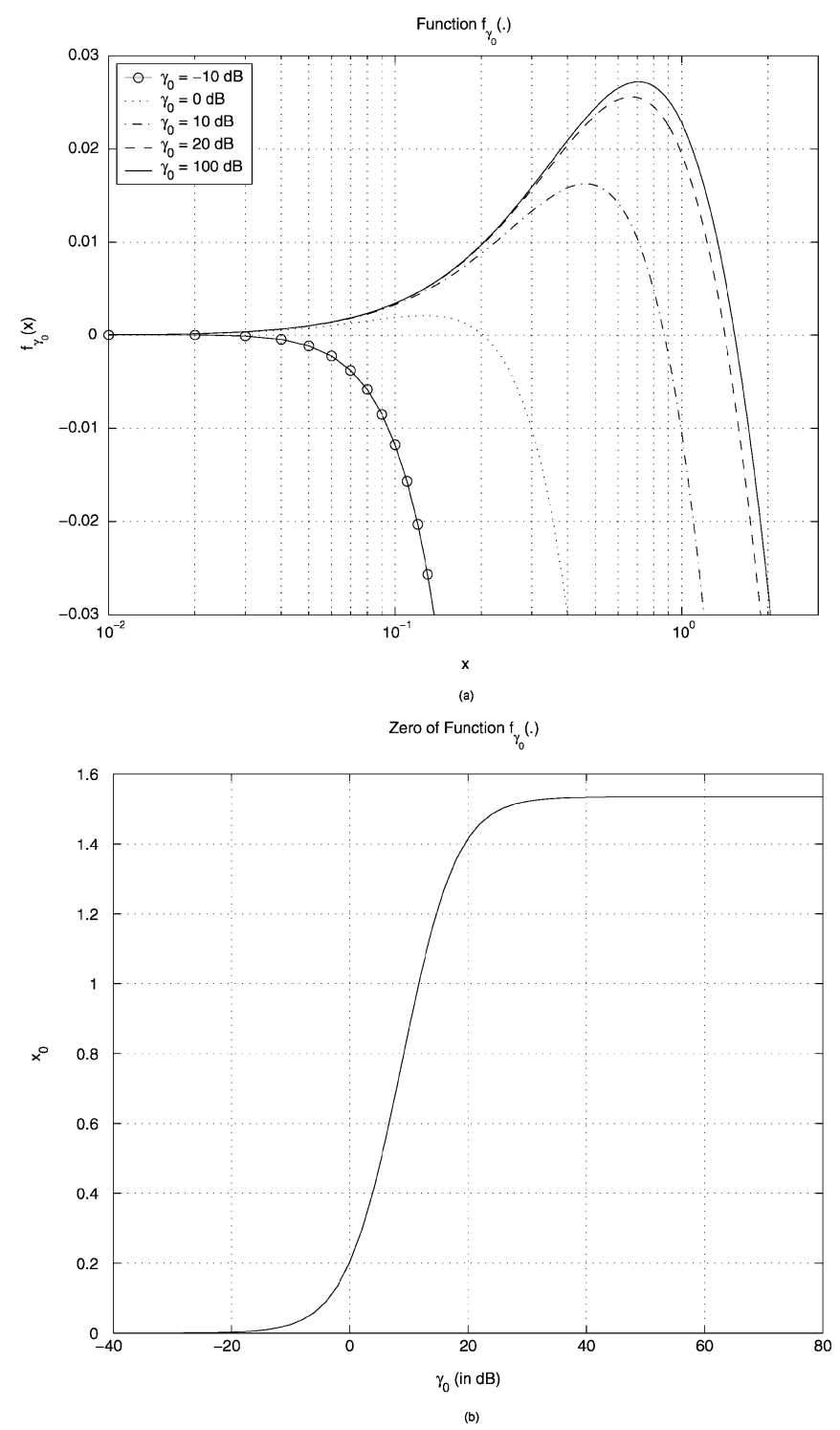

Fig. 5. Sensor system-size optimization under a total power constraint. $\gamma_{c}=$ $20 \mathrm{~dB}$. (a) Function $f_{\gamma_{0}}(\cdot)$. (b) Zero of the function $f_{\gamma_{0}}(\cdot)$.

This optimal number of sensors for a given total power constraint can be approximated as follows:

$$
n_{0} \approx \begin{cases}\frac{\gamma_{c}}{\tilde{x}_{0},} & \text { if } \gamma_{0} \gg 1 \\ \gamma_{c}, & \text { if } \gamma_{0} \ll 1\end{cases}
$$

where $\tilde{x}_{0} \approx 1.535$.

Proof: See Appendix IV.

The function $f_{\gamma_{0}}$ is shown on Fig. 5(a) as a parameterized plot. It is a well-behaved, smooth function with a unique zero. Moreover, as can be seen from Fig. 5(b), for both very small and very large values of $\gamma_{0}$ the zero of $f_{\gamma_{0}}$ converges to the limits specified in Proof of Proposition 6 (for medium values of $\gamma_{0}$, we need numerical methods to find the unique zero of function $\left.f_{\gamma_{0}}\right)$. In Fig. 6 we have shown the optimal number of sensor nodes for distributed detection of a stochastic signal under a total power constraint obtained via the exact solution to the zero of $f_{\gamma_{0}}$. Fig. 6 shows that indeed the asymptotic solutions given in

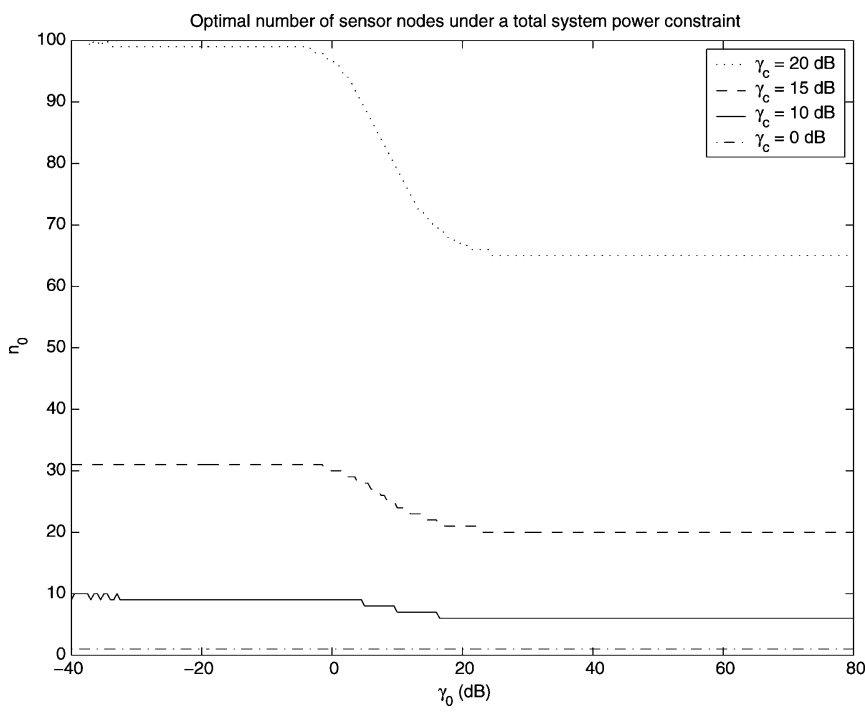

Fig. 6. Optimal number of sensor nodes as a function of the observation SNR for a given channel SNR. The system is under a global system power constraint with orthogonal sensor-to-fusion center communication.

(40) provide a very good approximation except for a small range of values for the observation SNR $\gamma_{0}$.

\section{B. Under Equicorrelated Signaling}

Let us now suppose that the signaling from sensors-to-fusion center is nonorthogonal with the normalized correlation matrix given by (15), where $|\rho|<1$ is the common cross-correlation between any pair of received signaling waveforms. The performance results derived in Section III-B still hold verbatim once we replace $\gamma$ by $\tilde{\gamma}$. These substitutions in the density of $T(\mathbf{r})$ given by (18) and in (19) specify the exact and the approximate fusion error probabilities, respectively, under a global power constraint $\tilde{P}_{0}$ with correlation matrix (15).

Similarly, corresponding to the eigenvalues $\lambda_{1}=\lambda_{2}=\cdots=$ $\lambda_{n-1}=1-\rho \triangleq \lambda_{a}$ and $\lambda_{n}=1+(n-1) \rho \triangleq \lambda_{b}$ of (15) we have $\sigma_{1, a}^{2}=\gamma_{0}(1+(n /(1-\rho) \tilde{\gamma}))^{-1}$ and $\sigma_{1, b}^{2}=$ $\gamma_{0}(1+(1 /(1-\rho / n+\rho) \tilde{\gamma}))^{-1}$. Substituting these in Proposition 4 gives the Chernoff and Bhattacharya error exponent in this case. Interestingly, in contrast to the orthogonal communication considered earlier, the optimal $s_{0}$ in this case is not monotonic in $n$. However, it can be shown that, for large $n$ it does converge to the following:

$$
\begin{aligned}
s_{0} & \longrightarrow 1+\frac{1}{\sigma_{1, b}^{2}}-\frac{1}{\log \left(1+\sigma_{1, b}^{2}\right)} \\
= & 1+\frac{1}{\gamma_{0}}+\frac{1}{\rho \gamma_{c}}\left(\frac{1}{2}+\frac{1}{\gamma_{0}}\right) \\
& -\frac{1}{\log \left(1+\frac{1}{\frac{1}{\gamma_{0}}+\frac{1}{\rho \gamma_{c}}\left(\frac{1}{2}+\frac{1}{\gamma_{0}}\right)}\right)} .
\end{aligned}
$$

Note that, in agreement with our earlier observations, above $s_{0}$ also satisfies $\lim _{\gamma_{0} \longrightarrow 0} s_{0}=(1 / 2)$.

A plot of the (tightest) Chernoff and Bhattacharya error exponents shows that, as with individual node power constraints, the performance degrades as $\rho$ increases. Moreover, again it can be shown that there is an optimal number of sensor nodes that results in the lowest upper bound for each $\rho$. As before, we base 


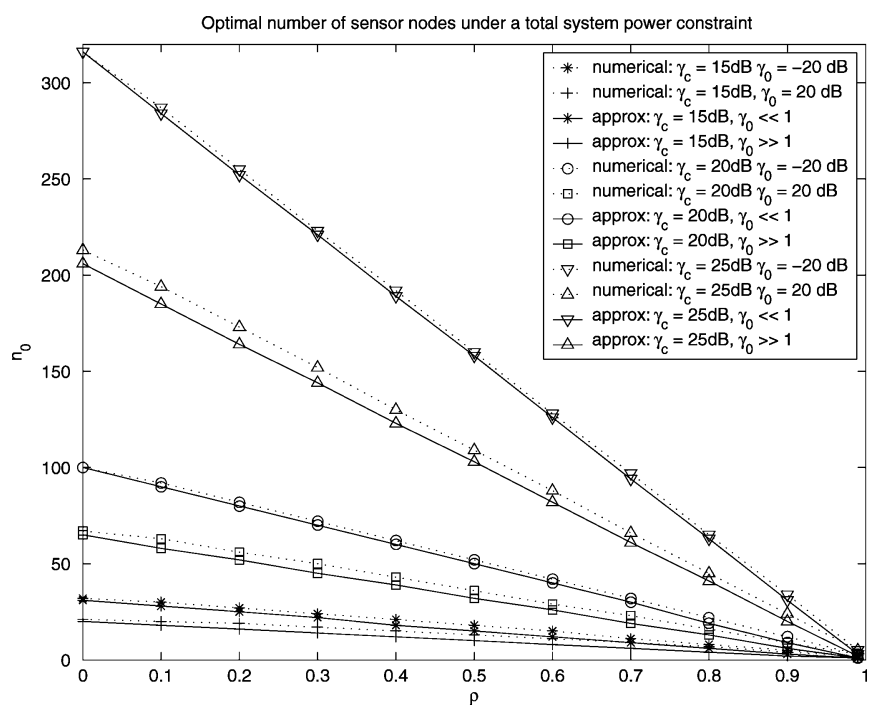

Fig. 7. Optimal number of sensor nodes as a function of the observation SNR for a given channel SNR. The system is under a global system power constraint with equicorrelated sensor-to-fusion center communication.

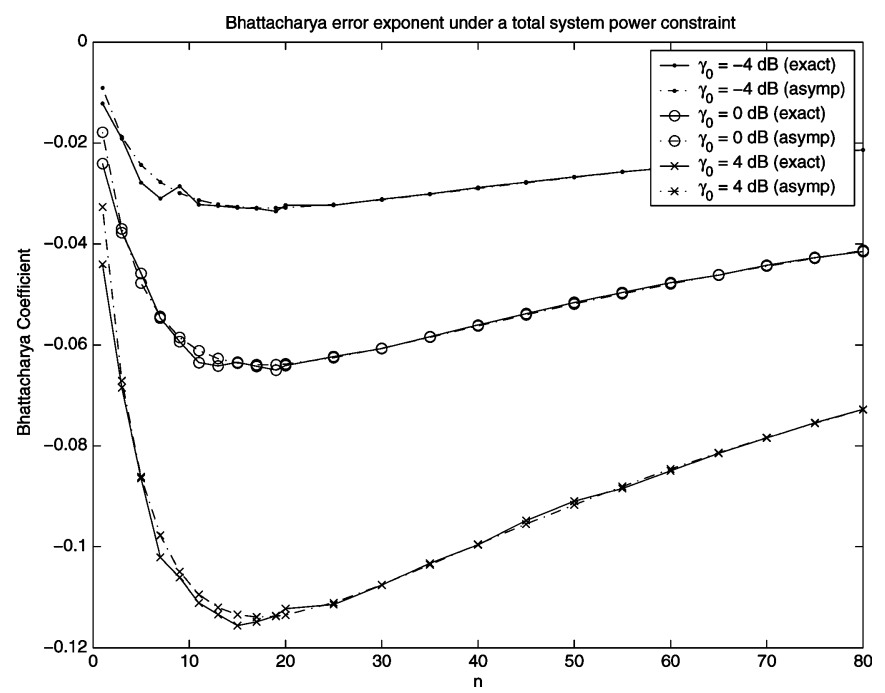

Fig. 8. The exact and large system approximation to the Bhattacharya error exponent with random signaling under a global power constraint.

our sensor system optimization on the Bharracharya error exponent. However, unlike in the case of orthogonal sensor-to-fusion center communication, a direct optimization of $\mu_{B}$ as a function of $n$ does not, in general, yield a closed-form expression. In Fig. 7 we have shown the numerically obtained optimal $n=n_{0}$ as a function of $\rho$ for different $\gamma_{0}$ and $\gamma_{c}$ values. As can be observed from Fig. 7, for a fixed $\gamma_{0}$ and $\gamma_{c}$, the optimal $n_{0}$ decreases as a function of $\rho$. An asymptotic expansion shows that optimal $n_{0}$ can be approximated as follows:

$$
n_{0} \approx \begin{cases}\frac{(1-\rho) \gamma_{c}}{\tilde{x}_{0}}, & \text { if } \gamma_{0} \gg 1 \\ (1-\rho) \gamma_{c}, & \text { if } \gamma_{0} \ll 1\end{cases}
$$

where $\tilde{x}_{0}$ is the unique zero of the function $\tilde{f}(\cdot)$ defined in (53) in the Proof of Proposition 6. In Fig. 7 we have also included the above approximations to optimal $n_{0}$. Note that, for a fixed $\gamma_{c}$ as $\rho$ increases, the approximations in (42) worsen. However,

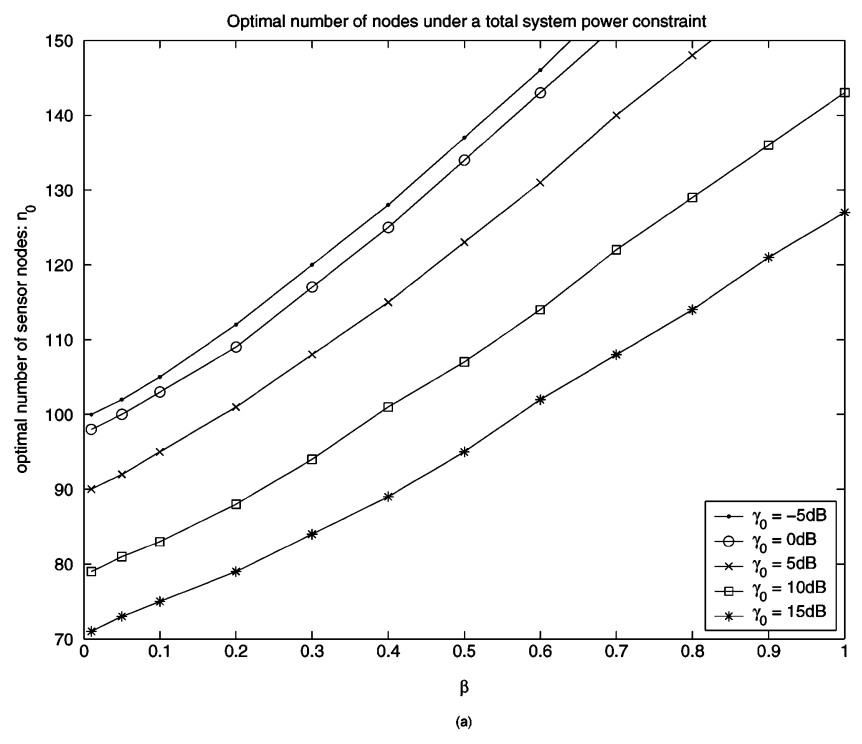

Optimal number of nodes under a total system power constraint

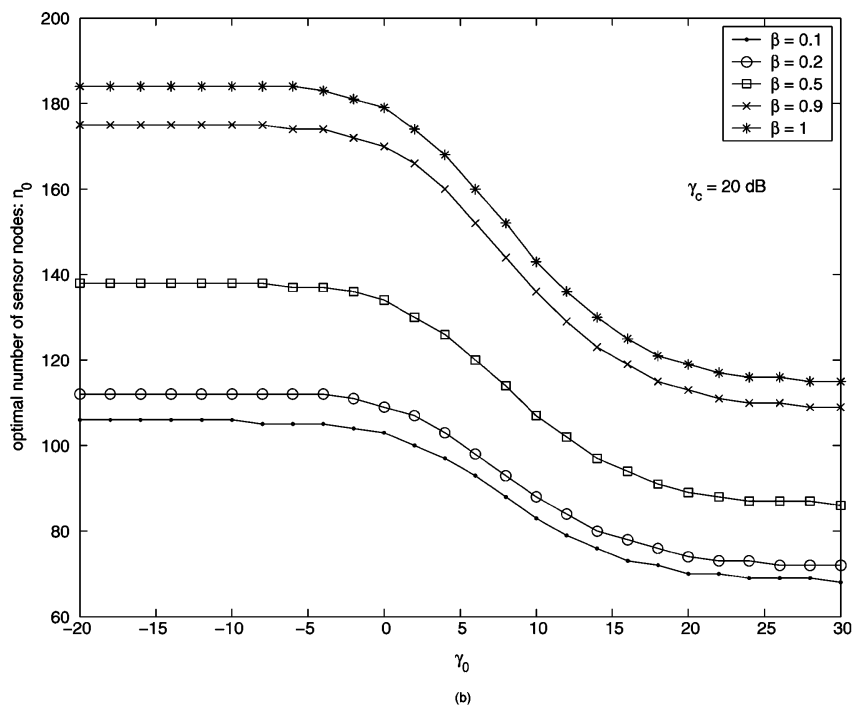

Fig. 9. Optimal sensor system size under a total power constraint. $\gamma_{c}=20 \mathrm{~dB}$ (a) For a fixed observation SNR $\gamma_{0}$. (b) For a fixed $\beta$.

as long as $(1-\rho) \gamma_{c}$ is not too small, (42) can be used as a good rule of thumb in optimizing a sensor system. It is interesting to note from (42) that $(1-\rho) \gamma_{c}$ again acts as the effective channel SNR. This confirms the fact that nonzero $\rho$ can only degrade the final fusion performance. In general, this is not true for the fusion of a deterministic signal with analog local processing as was shown in [14]. With a deterministic signal, at least when the system is perfectly synchronized, [14] showed that the nonzero $\rho$ can improve the fusion performance due to the effect of beam-forming. The reason is that, with a deterministic signal essentially all nodes transmit exactly the same information. In particular, when $\rho=1$ the signals from distributed nodes create a perfectly directed beam towards the fusion center thereby exploiting full coherent gain, whereas when $\rho=0$ the cooperative beam-forming gain is lost due to the use of orthogonal channels to send essentially the same information. In contrast, in the case of a random signal, the nonorthogonal signaling can never improve the final fusion performance as confirmed by the above results. 


\section{Under Random Signaling}

Suppose now that the elements of the signaling waveform $\mathbf{s}_{k}$ of the $k$ th node are iid, zero-mean random variables with $(1 / N)$ variances. For large $n$, the Bhattacharya error exponent corresponding to the fusion performance of a random signal still converges to (23) after the replacement of $\gamma$ by $\tilde{\gamma}$. As we have seen several times already the resulting performance however dramatically changes from that in individual node power constraint situation as can be seen from Fig. 8 for $\beta=1$. In contrast to what we saw under individual node power constraints, there is an optimal number of sensor nodes that results in the best possible fusion error probability. Moreover, as before, even for very small $n$ the asymptotic error exponent provides a tight approximation to the exact (random) Bhattacharya error exponent computed for a specific realization of the matrix $\mathbf{S}$.

A plot against $\beta$ will also show that as $\beta \longrightarrow 0$ the error exponent (23) converges to that of an orthogonal system $\mu_{B}^{0}$ as suggested by Corollary 1 . As can be seen from Fig. 9(a) the optimal number of nodes $n_{0}$ increases as $\beta$ increases for a fixed set of $\gamma_{c}$ and $\gamma_{0}$ whereas $n_{0}$ decreases as $\gamma_{0}$ increases for fixed $\beta$ and $\gamma_{c}$ as explicitly shown in Fig. 9(b). It is interesting to note that the dependance on $\gamma_{0}$ seen in Fig. 9(b) is the same as that observed in Fig. 6 for orthogonal signaling. In particular, it is easy to see that as $\beta \longrightarrow 0$, the optimal number of nodes in Fig. 9(b) for the case of random signaling indeed seems to agree with that shown in Fig. 6. This is of course a consequence of Corollary 1.

\section{CONCLUSION}

This paper analyzed the fusion performance of a stochastic Gaussian signal in a distributed sensor system under power and spectral constraints. Both average individual node power constraints as well as average total system power constraints were investigated under the assumption of analog relay-amplifier local processing. One of the main results is that in the case of a global average power constraint, there is an optimal number of local decisions that results in the best error performance at the fusion center, in contrast to the monotonic performance improvement observed in the decentralized detection of a deterministic signal (subjected to a global power constraint) as in [9]. Thus, there is a fundamental difference in the fusion performance behavior of deterministic and random signal distributed detection under a total system power constraint. Beyond this optimal value the performance degrades as the number of sensors is increased.

The fusion performance was investigated with: 1) orthogonal; 2) equicorrelated; and 3) random signaling waveforms for sensor-to-fusion center communication. Since only in certain situations Bayesian fusion performance can be derived in closed form, our analysis was based on the Chernoff and Bhattacharya upper bounds to the fusion error probability. We also proposed a sensor system optimization method based on Bhattacharya coefficient that leads to simple rules for determining the optimal number of nodes under a global average power constraint. The results showed that for a fixed observation quality $\mathrm{SNR} \gamma_{0}$ and a fixed global power constraint $\tilde{P}_{0}$, the optimal number of nodes $n_{0}$ monotonically increases with increasing spectral efficiency parameter $\beta$. On the other hand, for a fixed spectral efficiency level $\beta$ and a fixed $\tilde{P}_{0}$, optimal $n_{0}$ decreases as $\gamma_{0}$ increases. We also showed that nonorthogonal signaling in general leads to degraded fusion performance. Possible future work includes, obtaining closed-form expressions for the optimal sensor network size $n_{0}$ in random signaling case, nonuniform power allocation across the sensors and consideration of other local processing schemes (in particular, quantized decisions).

\section{APPENDIX I}

DERIVATION OF (4)

It is easily seen that the likelihood ratio can be written as

$$
\begin{aligned}
\mathcal{L}(\mathbf{r})= & \left(\frac{\left|\Sigma_{r}\right|}{\left|g^{2} \sigma_{x}^{2} \mathbf{R}^{2}+\Sigma_{r}\right|}\right)^{\frac{1}{2}} \\
& \times \exp \left(\frac{1}{2} \mathbf{r}^{T}\left(\Sigma_{r}^{-1}-\left(g^{2} \sigma_{x}^{2} \mathbf{R}^{2}+\Sigma_{r}\right)^{-1}\right) \mathbf{r}\right)
\end{aligned}
$$

Noting that $\xi_{k} \mathrm{~s}$ form a complete, orthonormal basis for $\mathbb{R}^{n}$ we have that $\mathbf{R}^{2}=\sum_{k=1}^{n} \lambda_{k}^{2} \xi_{k} \xi_{k}^{T}$ and $\Sigma_{r}=\sum_{k=1}^{n}\left(g^{2} \sigma_{v}^{2} \lambda_{k}+\right.$ $\left.\sigma_{w}^{2}\right) \lambda_{k} \boldsymbol{\xi}_{k} \boldsymbol{\xi}_{k}^{T}$. Using these in (43) leads to (44), shown at the bottom of the page. If we define a new set of random variables, for $k=1, \ldots, n$, as

$$
Y_{k}=\sqrt{\frac{g^{2} \sigma_{x}^{2}}{\left(g^{2} \sigma_{v}^{2} \lambda_{k}+\sigma_{w}^{2}\right)\left(g^{2}\left(\sigma_{x}^{2}+\sigma_{v}^{2}\right) \lambda_{k}+\sigma_{w}^{2}\right)}} \boldsymbol{\xi}_{k}^{T} \mathbf{r}
$$

then, by virtue of the orthonormality of $\mathbf{v}_{k} \mathrm{~s}$, it is easy to show that $Y_{k} \mathrm{~s}$ are a set of zero-mean and independent Gaussian random variables under both hypotheses that is equivalent to the original statistic r. Substitution of (45) in (44) leads to (4). From (45) it is easy to verify that the variances $\sigma_{j, k}^{2}$, for $j=0,1$ and $k=1, \ldots, n$, are given by (5).

\section{APPENDIX II}

\section{The Proof of Proposition 1}

Let us denote the common variance of $Y_{1}, \ldots, Y_{n-1}$ under $H_{j}$ as $\sigma_{j, a}^{2}$ and that of $Y_{n}$ as $\sigma_{j, b}^{2}$, where, from (5)

$$
\sigma_{j, a}^{2}= \begin{cases}\frac{\gamma_{0}}{1+\gamma_{0}+\frac{1}{\gamma \lambda_{a}}}, & \text { if } j=0 \\ \frac{\gamma_{0}}{1+\frac{1}{\gamma \lambda_{a}},}, & \text { if } j=1\end{cases}
$$

and $\sigma_{j, b}^{2}$ is defined similarly by replacing $\lambda_{a} \mathrm{~s}$ in (46) by $\lambda_{b}$. Let us defined $Z_{a}=\sum_{k=1}^{n-1} Y_{k}^{2}$ and $Z_{b}=Y_{n}^{2}$ such that $T(\mathbf{r})=Z_{a}+Z_{b}$. Then it follows that under $H_{j}, Z_{a} \sim$

$$
\mathcal{L}(\mathbf{r})=\exp \left(\frac{1}{2} \sum_{k=1}^{n} \frac{g^{2} \sigma_{x}^{2}}{\left(g^{2} \sigma_{v}^{2} \lambda_{k}+\sigma_{w}^{2}\right)\left(g^{2}\left(\sigma_{x}^{2}+\sigma_{v}^{2}\right) \lambda_{k}+\sigma_{w}^{2}\right)}\left|\boldsymbol{\xi}_{k}^{T} \mathbf{r}\right|^{2}\right) \prod_{k=1}^{n}\left(\frac{g^{2} \sigma_{v}^{2} \lambda_{k}+\sigma_{w}^{2}}{g^{2}\left(\sigma_{x}^{2}+\sigma_{v}^{2}\right) \lambda_{k}+\sigma_{w}^{2}}\right)^{\frac{1}{2}}
$$


$G\left((n-1 / 2) ;\left(1 / 2 \sigma_{j, a}^{2}\right)\right)$ and $Z_{b} \sim G\left((1 / 2) ;\left(1 / 2 \sigma_{j, b}^{2}\right)\right)$. The pdf of the decision statistic $T$ can then shown to be

$$
\begin{aligned}
p_{T}\left(t \mid H_{j}\right)= & \frac{\exp \left(\frac{-t}{2 \sigma_{j, b}^{2}}\right)}{\Gamma\left(\frac{n-1}{2}\right)\left(2 \sigma_{j, a}^{2}\right)^{\frac{n-1}{2}}\left(2 \pi \sigma_{j, b}^{2}\right)^{\frac{1}{2}}} \\
& \times \int_{0}^{t} e^{-z\left(\frac{1}{2 \sigma_{j, a}^{2}}-\frac{1}{2 \sigma_{j, b}^{2}}\right)} z^{\frac{n-3}{2}}(t-z)^{-\frac{1}{2}} d z .
\end{aligned}
$$

We obtain (18) from (47) by making use of the integral tables from [22] followed by the substitutions $B(x, y)=(\Gamma(x) \Gamma(y) / \Gamma(x+y))$ and $\Gamma(1 / 2)=\sqrt{\pi}$ where $B(x, y)$ is the beta function.

For large $n$ we may write ${ }_{1} F_{1}((n-1 / 2) ;(n / 2) ; t) \approx$ $\sum_{k=0}^{\infty}\left(t^{k} / k !\right)=e^{t}$. Using this in (18), followed by proper normalization (required for any valid pdf) shows that in the case of large $n$, we may approximate $p_{T}\left(t \mid H_{j}\right) \approx$ $G\left((n / 2) ;\left(1 / 2 \sigma_{j, a}^{2}\right)\right)$. This leads to approximate error probabilities as specified by (19) for $n \gg 1$.

\section{APPENDIX III}

\section{PROOF OF PROPOSITION 4}

Denote the empirical distribution of the eigenvalues of $\mathbf{R}$ by $F_{n}(\lambda)=(1 / n) \sum_{k=1}^{n} \mathcal{I}_{\left\{\lambda_{k} \leq \lambda\right\}}$, where $\mathcal{I}_{\mathcal{A}}$ is the indicator function of set $\mathcal{A}$. Notice that, for any finite $n, F_{n}(\lambda)$ is a random distribution. However, under the assumption of iid random elements of the matrix $\mathbf{S}$ with mean zero and variance $(1 / N)$, as the matrix dimensions $n$ and $N$ grows without bound such that $\lim _{n \longrightarrow \infty}(n / N)=\beta$, it is known that $F_{n}(\lambda)$ converges almost surely to a deterministic distribution $F(\lambda)$ with the corresponding density $f(\lambda)$ that only depends on the ratio $\beta$ as shown [20], [21], [23]: [see (48) at the bottom of the page], where $\delta(\lambda)$ is the Dirac-delta function, $[\lambda]^{+}=\max \{0, \lambda\}$, and $a(\beta)$ and $b(\beta)$ are defined in (25).

By substituting for $\sigma_{1, k}^{2}$ from (5) in the definition in (9), followed by the application of the definition of empirical eigenvalue distribution $F_{n}(\lambda)$ we can then show that

$$
\begin{aligned}
\mu_{B}= & \frac{n}{2} \int_{0}^{\infty}\left[\frac{1}{2} \log (1+\gamma \lambda)\right. \\
& +\frac{1}{2} \log \left(1+\left(1+\gamma_{0}\right) \gamma \lambda\right) \\
& \left.-\log \left(1+\left(1+\frac{\gamma_{0}}{2}\right) \gamma \lambda\right)\right] d F_{n}(\lambda) .
\end{aligned}
$$

Then by the convergence of the empirical eigenvalue distribution to a deterministic distribution with density $f(\lambda)$, in the limit of a large system the normalized Bhattacharya error exponent converges almost surely to

$$
\begin{aligned}
\frac{\mu_{B}}{n} \stackrel{\text { a.s. }}{\longrightarrow} & \frac{1}{2} \int_{0}^{\infty}\left[\frac{1}{2} \log (1+\gamma \lambda)\right. \\
& +\frac{1}{2} \log \left(1+\left(1+\gamma_{0}\right) \gamma \lambda\right) \\
& \left.-\log \left(1+\left(1+\frac{\gamma_{0}}{2}\right) \gamma \lambda\right)\right] f(\lambda) d \lambda .
\end{aligned}
$$

Now, from the results in [24] one can verify the following integral identity

$$
\begin{aligned}
\int_{0}^{\infty} & \log (1+x \lambda) f(\lambda) d \lambda \\
= & \log \left(1+x-\frac{1}{4} \psi(x, \beta)\right) \\
& +\frac{1}{\beta} \log \left(1+x \beta-\frac{1}{4} \psi(x, \beta)\right)-\frac{1}{4 x \beta} \psi(x, \beta)
\end{aligned}
$$

that is valid for any $\beta=(n / N)>0$, where function $\psi(x, \lambda)$ is as defined in (24). Substitution of (51) in (50) results in the asymptotic expression for the Bhattacharya error exponent as given in (23) of the Proposition 4. The large $\gamma_{0}$ asymptotic (26) follows by applying standard limit theorems.

\section{APPENDIX IV}

PROOF OF PROPOSITION 6

We obtain (38) by differentiating (37) with respect to $n$ and letting

$$
x=\frac{\sigma_{1}^{2}}{2}=\frac{\gamma_{0}}{2\left(1+\frac{n}{\gamma_{c}}\left(1+\frac{\gamma_{0}}{2}\right)\right)} .
$$

For $\gamma_{0} \gg 1$, using of standard limit techniques it is straightforward to see that (39) reduces to

$$
\tilde{f}(x)=\log \frac{\sqrt{1+2 x}}{1+x}+\frac{x^{2}}{(1+x)(1+2 x)}
$$

Thus, for $\gamma_{0} \gg 1$, the optimal $x_{0}$ converges to $\tilde{x}_{0}$, the zero of $\tilde{f}(\cdot)$ in (53). Substituting $x_{0}=\tilde{x}_{0}$ in (38) and using the fact that $\gamma_{0} \gg 1$ gives $n_{0} \approx\left(\gamma_{c} / \tilde{x}\right)$. Numerical techniques can be used to show that the unique zero $\tilde{x}_{0}$ of $\tilde{f}(\cdot)$ is $\tilde{x}_{0} \approx 1.535$.

Since $\gamma_{0} \ll 1$ implies that $x \ll 1$, using the fact that $\log (1+x) \approx x-(1 / 2) x^{2}$ for small $x$, we have that $\log (\sqrt{1+2 x} / 1+x) \approx-\left(x^{2} / 2\right)$. Substitution of (39) gives

$$
f(\lambda)= \begin{cases}{\left[1-\frac{1}{\beta}\right]^{+} \delta(\lambda)+\frac{\sqrt{(\lambda-a(\beta))(b(\beta)-\lambda)}}{2 \pi \beta \lambda}} & \text { if } a(\beta) \leq \lambda \leq b(\beta) \\ 0 & \text { otherwise }\end{cases}
$$


the solution $x_{0} \approx\left(\gamma_{0} / 4\right)$ which, when used in (38), leads to $n_{0} \approx \gamma_{c}$ for $\gamma_{0} \ll 1$.

\section{REFERENCES}

[1] R. R. Tenney and N. R. Sandell Jr., "Detection with distributed sensors," IEEE Trans. Aerosp. Electron. Syst., vol. AES-17, no. 4, pp. 501-510, Jul. 1981.

[2] P. K. Varshney, Distributed Detection and Data Fusion. New York: Springer-Verlag, 1996.

[3] R. Viswanathan and P. K. Varshney, "Distributed detection with multiple sensors: Part I-Fundamentals," Proc. IEEE, vol. 85, no. 1, pp. 54-63, Jan. 1997.

[4] J. N. Tsistsiklis, "Decentralized detection," in Advances in Statistical Signal Processing, H. V. Poor and J. B. Thomas, Eds. Greenwich, CT: JAI Press, 1993, pp. 297-344, Proc. Adv. Statist. Signal Process., Signal Detect.

[5] R. S. Blum, S. A. Kassam, and H. V. Poor, "Distributed detection with multiple sensors: Part II-Advanced topics," Proc. IEEE, vol. 85, no. 1, pp. 64-79, Jan. 1997.

[6] J. Chamberland and V. V. Veeravalli, "Asymptotic results for decentralized detection in power constrained wireless sensor networks," IEEE J. Sel. Areas Commun., vol. 22, no. 6, pp. 1007-1015, Aug. 2004.

[7] _ - "Decentralized detection in wireless sensor systems with dependent observations," in Proc. 2nd Int. Conf. Comput., Commun. Contr. Technol., Austin, TX, Aug. 2004.

[8] S. Appadwedula, V. V. Veeravalli, and D. L. Jones, "Energy-efficient detection in sensor networks," IEEE J. Sel. Areas Commun., vol. 23, pp. 693-702, Apr. 2005.

[9] S. K. Jayaweera, "Large system decentralized detection performance under communication constraints," IEEE Commun. Lett., vol. 9, pp. 769-771, Sep. 2005.

[10] S. K. Jayaweera, "Decentralized detection of stochastic signals in power-constrained sensor networks," presented at the IEEE Workshop on Signal Process. Adv. Wireless Commun. (SPAWC), New York, Jun. 2005.

[11] _ _ "Sensor system optimization for Bayesian fusion of distributed stochastic signals under resource constraints," in IEEE Int. Conf. Acoust., Speech Signal Process. (ICASSP'06), Toulouse, France, May 2006, pp. IV-149-IV-152.

[12] R. Niu, B. Chen, and P. K. Varshney, "Decision fusion rules in wireless sensor networks using fading channel statistics," in Proc. Conf. Inf. Sci. Syst., Mar. 2003, Johns Hopkins Univ..

[13] C. W. Helstrom, Statistical Theory of Signal Detection, 2nd ed. Oxford, U.K.: Pergamon, 1968.

[14] K. Altarazi, S. K. Jayaweera, and V. Aravinthan, "Performance of decentralized detection in a resource-constrained sensor network," in Proc. 39th Annu. Asilomar Conf. Signal, Syst. Comput., Pacific Grove, CA, Nov. 2005, pp. 437-441.
[15] H. Ochiai, P. Mitran, H. V. Poor, and V. Tarokh, "Collaborative beamforming for distributed wireless ad hoc sensor networks," IEEE Trans. Signal Process., vol. 53, no. 11, pp. 4110-4124, Nov. 2005.

[16] H. V. Poor, An Introduction to Signal Detection and Estimation. New York: Springer-Verlag, 1994.

[17] J. Chamberland and V. Veeravalli, "Decentralized detection in sensor networks," IEEE Trans. Signal Process., vol. 51, no. 2, pp. 407-416, Feb. 2003.

[18] R. C. Dixon, Spread Spectrum Systems with Commercial Applications. New York: Wiley, 1994.

[19] G. N. Karystinos and D. A. Pados, "On the epmirical distribution eigenvalues of a class of large dimensional random matrices," IEEE Trans. Inf. Theory, vol. 51, no. 1, pp. 348-355, Jan. 2005.

[20] J. W. Silverstein and Z. D. Bai, "On the epmirical distribution eigenvalues of a class of large dimensional random matrices," J. Mult. Anal., vol. 54, pp. 175-192, 1995.

[21] J. W. Silverstein, "Strong convergence of the epmirical distribution eigenvalues of large dimensional random matrices," J. Mult. Anal., vol. 55, pp. 331-339, 1995.

[22] I. S. Gradshteyn and I. M. Ryzhik, Tables of Integrals, Series, and Products. New York: Academic, 1965.

[23] S. Verdu, Multiuser Detection. Cambridge, U.K.: Cambridge Univ. Press, 1998.

[24] S. Verdu and S. Shamai, "Spectral efficiency of CDMA with random spreading," IEEE Trans. Inf. Theory, vol. 45, no. 2, pp. 622-640, 1999.

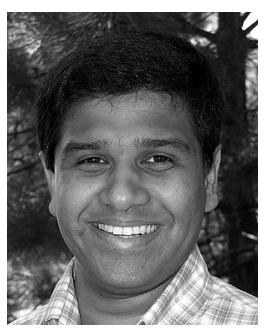

Sudharman K. Jayaweera (S'00-M'04) received the B.E. degree in electrical and electronic engineering with First Class Honors from the University of Melbourne, Australia, in 1997 and the M.A. and $\mathrm{Ph} . \mathrm{D}$. degrees in electrical engineering from Princeton University, Princeton, NJ, in 2001 and 2003, respectively.

He is currently an Assistant Professor of electrical engineering at the Department of Electrical and Computer Engineering, University of New Mexico, Albuquerque. From 2003 to 2006, he was an Assistant Professor of electrical engineering at the Department of Electrical and Computer Engineering, Wichita State University, KS. From 1998 to August 1999, he was with the US Wireless Corporation, San Ramon, CA, as a member of the Wireless Signal Processing Algorithms Development Group, where he was involved in developing wireless geolocation and tracking algorithms for the company's proprietary RadioCameraTM technology. His current research interests include wireless communications, information theory, statistical signal processing, and wireless sensor networks.

Dr. Jayaweera is currently an Associate Editor of EURASIP Journal of Applied Signal Processing. 